\title{
DÜBLIN
}

Technological University Dublin

ARROW@TU Dublin

Articles

School of Mathematics

2020-06-01

\section{On the Socles of Fully Inert Subgroups of Abelian p-Groups}

Andrey R. Chekhlov

Tomsk State University

Peter V. Danchev

Bulgarian Academy of Sciences

Brendan Goldsmith

Technological University Dublin, brendan.goldsmith@tudublin.ie

Follow this and additional works at: https://arrow.tudublin.ie/scschmatart

Part of the Applied Mathematics Commons, and the Mathematics Commons

\section{Recommended Citation}

Chekhlov, A., Danchev, P.V. \& Goldsmith, B. (2020). On the socles of fully inert subgroups of abelian pgroups. Mediterranean Journal of Mathematics, ahead of print.

This Article is brought to you for free and open access by the School of Mathematics at ARROW@TU Dublin. It has been accepted for inclusion in Articles by an authorized administrator of ARROW@TU Dublin. For more information, please contact arrow.admin@tudublin.ie, aisling.coyne@tudublin.ie,gerard.connolly@tudublin.ie.

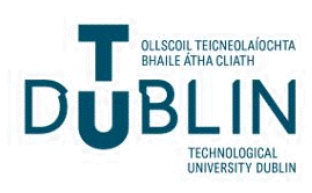




\title{
ON THE SOCLES OF FULLY INERT SUBGROUPS OF ABELIAN $p$-GROUPS
}

\author{
ANDREY R. CHEKHLOV, PETER V. DANCHEV, AND BRENDAN GOLDSMITH
}

\begin{abstract}
We define the so-called fully inert socle-regular and weakly fully inert socle-regular Abelian p-groups and study them with respect to certain of their numerous interesting properties. For instance, we prove that in the case of groups of length $\omega$ these two group classes coincide but that in the case of groups of length $\omega+1$ they differ. Some structural and characterization results are also obtained.

The work generalizes concepts which have been of interest recently in the theory of entropy in algebra and builds on recent investigations by the second and third named authors in Arch. Math. Basel (2009) and J. Algebra (2010).
\end{abstract}

\section{InTRODUCTION AND BACKGROUND}

Some years ago the second and third named authors introduced a notion called socle-regularity in [4] and [5], which turned out to be an interesting and useful concept. The underlying idea was to circumvent the difficulties in classifying fully invariant subgroups of Abelian p-groups by looking at their socles rather than the full subgroup. Hence a $p$-group $G$ was said to be socle-regular if, given any fully invariant subgroup $H$ of $G$, there is an ordinal $\alpha$, depending on $H$, with $H[p]=$ $p^{\alpha} G[p]$. Recently there has been a great deal of interest, arising primarily from considerations of various types of entropy, in another class of subgroups of $p$-groups (and indeed more generally in arbitrary commutative and non-commutative groups), the fully inert subgroups. Recall that the subgroups $H, K$ of a group $G$ are said to be commensurable if the intersection $H \cap K$ has finite index simultaneously in $H$ and in $K$; this is conventionally denoted $H \sim K$ and is known to be an equivalence relation.

2010 Mathematics Subject Classification. 20K10.

Key words and phrases. socle-regular groups, fully inert subgroups, fully inert socle-regular groups, weakly fully inert socle-regular groups. 
Then the subgroup $H$ of a group $G$ is said to be fully inert in $G$ if the factor-group $(H+\varphi(H)) / H$ is finite, i.e., $H$ is commensurable with $H+\varphi(H)$ for any $\varphi \in \mathrm{E}(G)$, the endomorphism ring of $G$; in particular, if $\varphi(H) \subseteq H$, the subgroup $H$ is known as fully invariant. It is also known, and easy to prove, that the sum and intersection of a finite number of fully inert subgroups are again fully inert and hence the socle $H[p]$ of a fully inert subgroup $H$ of the group $G$ is also fully inert in $G$. Fully inert subgroups of a group are, in some sense, not too far from fully invariant subgroups. Thus the issue of finding a natural generalization of the notion of socle-regularity arises for these subgroups. Three natural possibilities present themselves:

- require for all fully inert subgroups $H$ of $G$ the existence of an ordinal $\alpha$, depending on $H$, such that $H[p]=p^{\alpha} G[p]$;

- require for all fully inert subgroups $H$ of $G$ the existence of an ordinal $\alpha$, depending on $H$, such that $H[p] \sim p^{\alpha} G[p]$;

A weaker alternative would be:

- require for all fully inert subgroups $H$ of $G$ the existence of an ordinal $\alpha$, depending on $H$, such that $H[p] \cap p^{\alpha} G[p]$ is of finite index in $p^{\alpha} G[p]$.

We will show in Section 5 below that the first possibility is so restrictive that it is of little interest and we will not assign any name to this concept. On the other hand, the remaining two concepts are interesting and with suitable modifications, they lead to the following definition:

Definition 1.1. An Abelian p-group $G$ is said to be fully inert socle-regular if, for all infinite fully inert subgroups $H$ of $G$, there exists an ordinal $\alpha$, depending on $H$, such that $H[p] \sim p^{\alpha} G[p]$; alternatively $G$ is said to be weakly fully inert socle-regular if, for all infinite fully inert subgroups $H$ of $G$, there exists an ordinal $\alpha$, depending on $H$, such that $p^{\alpha} G \neq 0$ and $H[p] \cap p^{\alpha} G[p]$ is of finite index in $p^{\alpha} G[p]$.

Note that the restriction to infinite fully inert subgroups in the definition of fully inert socle-regularity is not restrictive: if $H$ is finite then $H \sim p^{\ell(G)} G=\{0\}$, where $\ell(G)$ denotes the length of $G$. However, the situation in weak fully inert socleregularity is more complicated and if one allows the choice $\alpha=\ell(G)$ then every 
group would be weakly fully inert socle-regular. Hence our restriction to infinite fully inert subgroups in both cases.

Clearly a fully inert socle-regular group is weakly fully inert socle-regular but we shall show by an example that the converse does not hold.

Throughout all groups considered, unless specified to the contrary, are reduced Abelian $p$-groups, where $p$ is a fixed but arbitrary prime. Almost all our terminology and notations are standard and follow those from [8] and [9]. For instance, for any prime $p$, the symbol $G\left[p^{n}\right]=\left\{g \in G: p^{n} g=0\right\}$ denotes the $p^{n}$-socle of the group $G$, and the symbol $p^{n} G=\left\{p^{n} g: g \in G\right\}$ denotes the $n$-th power subgroup of $G$, where $n \in \mathbb{N}$. Inductively, for any ordinal $\alpha, p^{\alpha} G=p\left(p^{\alpha-1} G\right)$ when $\alpha-1$ exists or $p^{\alpha} G=\cap_{\beta<\alpha} p^{\beta} G$ otherwise; recall that a $p$-group $G$ is said to be separable if $p^{\omega} G=\{0\}$. Since all groups $G$ considered are reduced by assumption, there exists a least integer $\tau$ such that $p^{\tau} G=0$. In this terminology our definition of weakly fully inert socle-regularity requires that the ordinal $\alpha$ is strictly less than the length of the group, $\alpha<\tau$.

We can see immediately that the class of weakly fully inert socle-regular groups is large. Consider the following ad hoc definition.

Definition 1.2. A reduced $p$-group of infinite length $\tau$ is said to be broad if, for all $\alpha<\tau, p^{\alpha} G$ is infinite.

Our first observation is the simple: a reduced $p$-group $G$ which is not broad is weakly fully inert socle-regular: for if $\alpha<\tau$ is such that $p^{\alpha} G$ is finite (and necessarily nonzero by the definition of length), then for any infinite fully inert subgroup $H$ of $G$ we have that $H \cap p^{\alpha} G[p]$ is finite (but possibly zero) and hence is commensurable with the finite subgroup $p^{\alpha} G[p]$.

Example 1.3. If $\sigma$ is not a limit ordinal, then the generalized Prüfer group $H_{\sigma}$ is weakly fully inert socle-regular.

The concept of broadness is, of course, mainly of use in dealing with non-separable groups. Surprisingly, separable groups are a great deal more complicated than one 
might expect given that it is easy to show (see, for example, [4]) that all such groups are socle-regular. Our next result is a useful way of establishing the existence of fully inert (and hence weakly fully inert) separable socle-regular groups of arbitrary cardinality.

Proposition 1.4. If $G$ is a reduced p-group with the property that every fully inert subgroup of $G$ is commensurable with a fully invariant subgroup of $G$, then if $G$ is socle-regular, it is also fully inert socle-regular.

Proof. Suppose that $H$ is fully inert in $G$, then by hypothesis $H \sim K$ for some fully invariant subgroup $K$ of $G$. It follows by standard properties of commensurability that $H \cap G[p] \sim K \cap G[p]$ and so $H[p] \sim K[p]$. However, as $G$ is socle-regular by hypothesis, we have that $K[p]=p^{\alpha} G[p]$ for some ordinal $\alpha$ and it follows that $H[p] \sim p^{\alpha} G[p]$. Thus $G$ is fully inert socle-regular as required.

An application of the principal results of [11] and [12] now gives:

Corollary 1.5. If $G$ is a direct sum of cyclic groups or a torsion-complete group, then $G$ is fully inert socle-regular (and thus also weakly fully inert socle-regular).

We shall show later that there is a further connection between fully inert socleregularity and the weak version of the concept when we are dealing with separable groups - see Corollary 4.10

We finish this section with two examples: the first is a separable group which is not even weakly fully inert socle-regular and the second is a weakly fully inert socleregular group which is not fully inert socle-regular.

Example 1.6. There exists a separable group which is not weakly fully inert socleregular.

Proof. Let $P$ be a standard $p$-group having endomorphism ring of the form $J_{p}$. $1_{P} \oplus E_{s}(P)$ - such groups have been constructed by various authors including Pierce [13], Corner [3] and Goldsmith [10]; note that the constructions all give groups of cardinality $2^{\aleph_{0}}$ but basic subgroups are countable. Then if $B$ is a basic subgroup of 
$P$, the argument of $[11$, Theorem 4.2$]$ shows that $B[p]$ is countable and fully inert in $P$. However, for any integer $n,\left|P / p^{n} P\right|=\left|B / p^{n} B\right|=\aleph_{0}$ and so $p^{n} P$ is uncountable and hence so too is $p^{n} P[p]$. Thus the countable group $B[p] \cap p^{n} P$ cannot be of finite index in $p^{n} P[p]$ for any integer $n$. Since $P$ is separable, $p^{\alpha} P=0$ for any $\alpha \geq \omega$ and so $P$ is not weakly fully inert socle-regular.

In fact, we can use a simple modification of Example 1.6 above to show that the class of fully inert socle-regular groups is strictly contained in the class of weakly fully inert socle-regular groups.

Example 1.7. There exists a weakly fully inert socle-regular group which is not fully inert socle-regular.

Proof. Our example utilises a construction made originally by Hausen and Johnson in [14]. Construct a group $G$ such that $p^{\omega} G=\mathbb{Z}(p)$ and $G / p^{\omega} G \cong P$, where $P$ is as in Example 1.6 - this is possible by a result of Kulikov (see [8, Theorem 76.1]) - and note that $G$ has basic subgroups isomorphic to the standard basic subgroups of $P$ (see Theorem 30.2 in [7]). Furthermore, it follows as in [14, Lemma 4.4], that the endomorphism ring of $G$ is precisely $J_{p} \cdot 1_{G} \oplus E_{s}(G)$.

Now the group $G$ is certainly not broad so it is weakly fully inert socle-regular. We claim that it is not fully inert socle-regular. Let $B^{\prime}$ be a basic subgroup of $G$ which is isomorphic to a basic subgroup $B$ of $P$. Then one claims that $B^{\prime}[p]$ is a fully inert subgroup of $G$. In fact, since every endomorphism of $G$ is of the form $\phi=r 1_{G}+\theta$, where $\theta$ is small, it follows from standard properties of small endomorphisms and from the fact that $B^{\prime}$ is standard, that the quotient $\left(B^{\prime}[p]+\phi\left(B^{\prime}[p]\right)\right) / B^{\prime}[p]$ is finite for all endomorphisms $\phi$ of $G$, i.e., $B^{\prime}[p]$ is really fully inert in $G$, as claimed. Now $B^{\prime}[p]$ is countably infinite while $p^{\omega} G[p]$ is finite, so $B^{\prime}[p]$ cannot be commensurable with $p^{\omega} G[p]$. Furthermore, for any finite $n, p^{n} G / p^{\omega} G \cong p^{n}\left(G / p^{\omega} G\right) \cong p^{n} P$ is uncountable, so that certainly $p^{n} G[p]$ is uncountable while $B^{\prime}[p]$ is countable, so that these groups cannot be commensurable. Hence $G$ is not fully inert socle-regular.

We have divided, somewhat arbitrarily, our main discussions into further five sections, the first of which contains preliminary material that will be used freely 
throughout the paper. In Section 3 we investigate the behavior of direct sums and direct summands of (weakly) fully inert socle-regular groups. This section includes an example of a weakly fully inert socle-regular group which has a summand that is not weakly fully inert socle-regular group and it also illustrates the complexity of the situation surrounding the direct sum of even just two such groups, see Theorem 3.9 and Proposition 3.11.

In Section 4 we consider subgroups of (weakly) fully inert socle-regular groups with some emphasis on non-separable groups. We show in Corollary 4.7 that totally projective groups (and in particular countable groups) of length not exceeding $\omega$. 2 are always weakly fully inert socle-regular and in Corollary 4.10 we have the interesting result that the concepts of weak fully inert socle-regularity and full inert socle-regularity coincide for separable groups; we also show that a group is fully inert socle-regular if, and only if, this is true of its finite index subgroups. In section 5 we show that the first proposed generalisation of socle-regularity above leads to the class of cyclic groups, and in the final sixth section we raise some left-open intriguing questions whose investigation may give further insight into the classes of (weakly) fully inert socle-regular groups.

\section{Preliminaries}

We begin by recalling some elementary facts relating to subgroups of finite index in a reduced $p$-group $G$; the proofs of the statements are straightforward and do not depend on full inertia of the subgroup $H$ of $G$.

- If $H \cap p^{\sigma} G[p]$ is of finite index in $p^{\sigma} G[p]$ for some ordinal $\sigma$, then $H \cap p^{\alpha} G[p]$ is of finite index in $p^{\alpha} G[p]$ for all $\alpha \geq \sigma$.

Consequently, if $G$ is a weakly fully inert socle-regular group, then there is a least

ordinal $\sigma_{0}=\sigma_{0}(H)$ such that $H \cap p^{\sigma_{0}} G[p]$ is of finite index in $p^{\sigma_{0}} G[p]$ and the same property holds for all $\alpha \geq \sigma_{0}$.

We remark that in general, it may happen that a group $G$, even a separable group, may have a fully inert subgroup $H$ which does not have this finite index property for 
any ordinal; it is easy to check that this happens for the Pierce group $P$ in Example 1.6.

In a similar vein we have:

- $H \cap p^{\delta} G[p]$ is of finite index in $H[p]$ for some $\delta$, then $H \cap p^{\beta} G[p]$ is of finite index in $H[p]$ for all $\beta \leq \delta$. So there exists a largest ordinal $\delta_{0}$ such $\delta_{0}=\delta_{0}(H)$ with this property; note that if $H$ is infinite, then $\delta_{0}<\ell(G)$. Notice that an ordinal $\delta$ always exists since $H \cap p^{0} G[p]=H[p]$.

Recall that it follows easily from the representation of endomorphisms of a direct sum that we always have:

- If $H$ is fully inert in the reduced $p$-group $G$, then $H \oplus H$ is fully inert in $G \oplus G$.

The following elementary result will be used frequently; it appears in various forms in, for example, [1, Lemma 3] or [12, Lemma 3.1].

Lemma 2.1. (i) If $A$ is a subgroup of the group $G$ having the property that every endomorphism of $A$ extends to an endomorphism of $G$, then if $H$ is fully inert in $G, H \cap A$ is fully inert in $A$.

(ii) If $H$ is a fully inert subgroup of $G=X \oplus Y$, then

$$
(H \cap X) \oplus(H \cap Y) \leq H \leq \pi_{X}(H) \oplus \pi_{Y}(H),
$$

where $\pi_{X}, \pi_{Y}$ are the canonical projections of $G$ onto $X, Y$ respectively. Furthermore, $(H \cap X) \oplus(H \cap Y) \sim H \sim \pi_{X}(H) \oplus \pi_{Y}(H)$ and if $\phi \in \operatorname{Hom}(Y, X)$, then $(H \cap X)+$ $\phi(H \cap Y) \sim(H \cap X)$.

The extension of Lemma 2.1 above to infinite decompositions requires care, since it can happen that a fully inert subgroup $H$ of a direct sum $\bigoplus_{I} G_{i}$ may have the property that $H \cap G_{i}=0$ for all $i \in I$ - for such an example see [12, Example 2.4]. The correct approach is to utilise the so-called box-like subgroups which is based on the work of Chekhlov [2] or [12].

Lemma 2.2. Let $H$ be a fully inert subgroup of the group $A=\bigoplus_{i \in I} G_{i}$, where the index set $I$ is infinite and let $\pi_{i}$ denote the canonical projections from $G$ onto $G_{i}$. Then $H$ is commensurable with $\bigoplus_{i \in I} \pi_{i}(H)$, the $\pi_{i}(H)$ are fully inert in $G_{i}$ and almost 
all $\pi_{i}(H)$ are fully invariant in $G_{i}$. Furthermore, there is a finite subset $S \subseteq I$ such that $\bigoplus_{i \in I \backslash S} \pi_{i}(H)$ is fully invariant in $\bigoplus_{i \in I \backslash S} G_{i}$.

Proof. It follows from [2, Lemma 4] that $H$ is commensurable with $\bigoplus_{i \in I} \pi_{i}(H)$; the rest of the assertions, except for the final two, follow from the previous lemma. So it remains to show that (i) almost all the $\pi_{i}(H)$ are fully invariant in $G_{i}$ and (ii) that there is a finite subset $I_{0} \subseteq I$ such that $\bigoplus_{i \in I \backslash I_{0}} \pi_{i}(H)$ is fully invariant in $\bigoplus_{i \in I \backslash I_{0}} G_{i}$. Firstly, assume for a contradiction that there is an infinite subset $S$ of $I$ such that $\pi_{s}(H)$ is not fully invariant in $G_{s}(s \in S)$. Then there exist endomorphisms $f_{s} \in$ $\operatorname{End}\left(G_{s}\right)(s \in S)$ such that $f_{s}\left(\pi_{s}(H)\right) \not \pi_{s}(H)$. Choose for each $s \in S$, an element $x_{s} \in H$ such that $f_{s}\left(\pi_{s}\left(x_{s}\right)\right) \notin \pi_{s}(H)$. Now $f_{s}\left(\pi_{s}\left(x_{s}\right)\right) \in G_{s}$, so $\pi_{s}\left(f_{s} \pi_{s}\left(x_{s}\right)\right)=$ $f_{s} \pi_{s}\left(x_{s}\right) \notin \pi_{s}(H)$, whence $f_{s} \pi_{s}\left(x_{s}\right) \notin H$. Now consider the elements $\left\{f_{s} \pi_{s}\left(x_{s}\right)+H\right.$ : $s \in S\}$ of the quotient $\left(f_{s} \pi_{s}(H)+H\right) / H$. Observe that the elements listed are distinct: if $f_{s} \pi_{s}\left(x_{s}\right)+H=f_{t} \pi_{t}\left(x_{t}\right)+H$ for $s \neq t \in S$, then $f_{s} \pi_{s}\left(x_{s}\right)-f_{t} \pi_{t}\left(x_{t}\right) \in H$ and applying the map $\pi_{s}$, we arrive at a contradiction $\pi_{s} f_{s} \pi_{s}\left(x_{s}\right)=f_{s} \pi_{s}\left(x_{s}\right) \in$ $\pi_{s}(H)$. Hence there are at most a finite number of indices $i \in I$ for which $\pi_{i}(H)$ is not fully invariant in $I$. Removing the groups $G_{i}$ corresponding to this finite set we arrive at an infinite subset $I_{0}$ of $I$ with $I \backslash I_{0}$ finite, such that $\pi_{I}(H)$ is fully invariant in $G_{i}$ for all $i \in I_{0}$.

For simplicity of notation we now write $H_{i}=\pi_{i}(H)$. Consider now the set $\mathcal{S}=$ $\left\{(i, j) \in I_{0} \times I_{0} \mid\right.$ there exists $\phi_{i j}: G_{j} \rightarrow G_{i}$ with $\left.\phi_{i j}\left(H_{j}\right) \nsubseteq H_{i}\right\}$. If the set $\mathcal{S}$ contains infinitely many distinct first co-ordinates $i$, then we can define a map $\psi: G \rightarrow G$ as follows: let $\psi\left(G_{j}\right)=\phi_{i j}\left(G_{j}\right)$ for each of the suitable $i$ and extend trivially on the remaining summands of $G$.

Now by our definition of $\mathcal{S}$ we know that there exists an element $x_{i} \in G_{i}$ say, with $x_{i} \in \phi_{i j}\left(H_{j}\right) \backslash H_{i}$. Consider the quotient $(\psi(H)+H) / H$; it contains the cosets $x_{i}+H$ which are all distinct, since if $x_{i}+H=x_{t}+H$ for some $t \neq i$, we have $x_{i}-x_{t} \in H$ and applying the map $\pi_{i}$ we get that $x_{i}=\pi_{i}\left(x_{i}-x_{t}\right) \in \pi_{i}(H)=H_{i}$. Thus the quotient $(\psi(H)+H) / H$ is infinite, contrary to $H$ being fully inert in $G$. Hence $\mathcal{S}$ has finitely many first co-ordinates $i$ and if we delete this finite set from $I_{0}$, we have 
a further infinite cofinite subset $I_{2}$ say, of $I$ where for all $\phi \in \operatorname{Hom}\left(G_{j}, G_{i}\right)$ we have $\phi\left(H_{j}\right) \leq H_{i}$.

Now work with the subset $I_{2}$ and reverse the roles of $i$ and $j$ in the previous argument. This will again gives us a finite number of indices $j$ to discard and the resulting infinite cofinite subset of $I$, say $I \backslash S$, will have the property that all 'off-diagonal' homomorphisms $\alpha \in \operatorname{Hom}\left(G_{i}, G_{j}\right)(i \neq j \in I \backslash S)$ have the property that $\alpha\left(H_{i}\right) \leq$ $H_{j}$. Since the $H_{i}(i \in I \backslash S)$ are also fully invariant in the corresponding $G_{i}$, we see that $\bigoplus_{i \in I \backslash S} \pi_{i}(H)$ is fully invariant in $\bigoplus_{i \in I \backslash S} G_{i}$.

The following technical result will also be used in several of our arguments, so we have isolated the core result into a simple proposition to be used as required.

Proposition 2.3. Suppose that $G$ is a reduced p-group and $A \leq G=G_{1} \oplus G_{2}$. If $A \cap p^{\alpha_{1}} G_{1}[p] \sim p^{\alpha_{1}} G_{1}[p]$ and $A \cap p^{\alpha_{2}} G_{2}[p] \sim p^{\alpha_{2}} G_{2}[p]$ for ordinals $\alpha_{1}, \alpha_{2}$ with $p^{\alpha_{1}} G_{1} \neq 0 \neq p^{\alpha_{2}} G_{2}$, then if $A \sim\left(A \cap G_{1}\right) \oplus\left(A \cap G_{2}\right)$, we have $A \cap p^{\beta} G[p] \sim p^{\beta} G[p]$, where $\beta=\max \left\{\alpha_{1}, \alpha_{2}\right\}$.

Proof. Since by hypothesis, $A \sim\left(A \cap G_{1}\right) \oplus\left(A \cap G_{2}\right)$, we have that $A \cap p^{\beta} G \sim$ $\left(\left(A \cap G_{1}\right) \oplus\left(A \cap G_{2}\right)\right) \cap p^{\beta} G$ and so $\left(A \cap p^{\beta} G\right)[p] \sim\left(A \cap p^{\beta} G_{1}\right)[p] \oplus\left(A \cap p^{\beta} G_{2}\right)[p] \sim$ $\left(A \cap p^{\alpha_{1}} G_{1}\right)[p] \oplus\left(A \cap p^{\alpha_{1}} G_{2}\right)[p] \sim p^{\alpha_{1}} G_{1} \oplus\left(A \cap p^{\alpha_{1}} G_{1}\right)[p]$, where, without loss of generality, we have assumed $\beta=\alpha_{1}$; note that $p^{\beta} G \neq 0$.

However, $\left(A \cap p^{\alpha_{2}} G_{2}\right)[p] \sim p^{\alpha_{2}} G_{2}[p]$ and, as $\alpha_{1} \geq \alpha_{2}$, we know from our earlier observation that we also have $\left(A \cap p^{\alpha_{1}} G_{2}\right)[p] \sim p^{\alpha_{1}} G_{2}[p]$, and so we get immediately the desired result, $\left(A \cap p^{\beta}\right) G[p] \sim p^{\beta} G[p]$.

We conclude this section with two further technical results which will be useful later.

Lemma 2.4. Suppose that $G$ is an infinite homocyclic group of exponent $n$ and $H$ is fully inert in $G$. Then either $H$ is finite, or there is a direct summand $C$ of $G$ of finite corank and $C[p]=H[p]$.

Proof. If $H$ is not finite then $H[p]$ is infinite and, since $G$ is a direct sum of cyclic groups, there is a pure subgroup $C$ of $G$ with $C[p]=H[p]$. In fact, since $G$ is bounded, $C$ is a direct summand of $G$. Let $G=C \oplus M$ and note that as $G$ is 
homocyclic, the summand $C$ can be expressed as $C=C_{1} \oplus C_{2}$, where $C_{1}=\bigoplus_{i=1}^{\infty}\left\langle c_{i}\right\rangle$ with each $c_{i}$ being of order $p^{n}$; observe that $M$ is again homocyclic of exponent $n$. Suppose for a contradiction that $M$ is infinite.

Choose a direct summand $M_{1}$ of $M$ with $M_{1}=\bigoplus_{i=1}^{\infty}\left\langle m_{i}\right\rangle$ with each $m_{i}$ being of order $p^{n}$. Now define $\phi: G \rightarrow G$ by setting $\phi$ to be zero on $C_{2} \oplus M$ and $\phi\left(c_{i}\right)=m_{i}$; this is a well-defined endomorphism of $G$ since $o\left(c_{i}\right)=o\left(m_{i}\right)$ for all $1 \leq i<\infty$.

Then $(H[p]+\phi(H[p])) / H[p]=\left(H[p]+\phi\left(C_{1}[p]\right)\right) / H[p] \cong \phi\left(C_{1}[p]\right) /\left(H[p] \cap \phi\left(C_{1}[p]\right)\right)=$ $M_{1}[p] /\left(H[p] \cap M_{1}[p]\right) \cong M_{1}[p]$ since $H[p] \cap M_{1}[p] \leq C[p] \cap M[p]=0$. This is impossible: $H[p]$ is fully inert in $G$ since $H$ is such and hence we must have $M_{1}[p]$ finite - contradiction since $M_{1}[p]=\bigoplus_{i=1}^{\infty}\left\langle p^{n-1} m_{i}\right\rangle$. This establishes the lemma.

Lemma 2.5. Suppose that $G$ is a reduced p-group and $G=A \oplus C \oplus M$, where $A, C$ are homogeneous groups of exponents $k, n$ respectively with $k<n$. If $H \leq G[p]$ is fully inert in $G$, then if $H \cap A$ is infinite, so too is $H \cap C$.

Proof. Let $(H \cap A)[p]=Y \oplus W$ where $Y$ is countably infinite. Then, as in the previous lemma, we can find a direct summand $X$ of $A$ with $X[p]=Y$. Assume now for a contradiction that $H \cap C$ is finite. Then $C[p]=(H \cap C)[p] \oplus L \oplus M$ where $L$ is a of countably infinite rank. Since $C$ is homocyclic there is a summand $Z$ of $C$ with $Z[p]=L$.

Write $X=\bigoplus_{i=1}^{\infty}\left\langle x_{i}\right\rangle$ with each $x_{i}$ being of order $p^{k}$ and let $Z=\bigoplus_{i=1}^{\infty}\left\langle z_{i}\right\rangle$ with each $z_{i}$ being of order $p^{n}$.

Now define $\phi: A \rightarrow C$ by setting $\phi$ to be zero on a complement of $X$ and $\phi\left(x_{i}\right)=$ $p^{n-k} z_{i}$; note that $\phi$ embeds $X$ into $Z$ and $\phi(Y)=L$. Clearly $\phi$ extends to an endomorphism of $G$ by trivial extension. However,

$$
(\phi(H)+H) / H \geq(\phi(X[p]) \oplus H) / H \cong \phi(X[p] /(H \cap \phi(X[p])) \cong L /(H \cap L)
$$

Since $H \cap L=\{0\}$, this gives the contradiction that $(\phi(H)+H) / H$ is infinite and the lemma is established. 


\section{Direct Sums and Direct Summands}

Our first result shows that weak full inert socle-regularity is preserved on taking finite direct sums of a single group. We present the result for the case of two copies of the group but it will be possible to extend the arguments to any finite number of copies utilizing Proposition 3.11 below; note that the proof of sufficiency below extends easily to an arbitrary finite integer.

Proposition 3.1. A group $G$ is weakly fully inert socle-regular if, and only if, the direct sum $A=G \oplus G$ is weakly fully inert socle-regular.

Proof. Assume $A$ is weakly fully inert socle-regular and let $H \leq G[p]$ be an infinite fully inert subgroup of $G$. Then $H \oplus H$ is fully inert infinite subgroup of $A$. So there exists an ordinal $\alpha$ with $p^{\alpha} A \neq 0$ and $\left((H \oplus H) \cap p^{\alpha} A\right)[p] \sim p^{\alpha} A[p]$; note that $p^{\alpha} G \neq 0$ follows.

However, it now follows that $\left(H \cap p^{\alpha} G[p]\right) \oplus\left(H \cap p^{\alpha} G[p]\right) \sim p^{\alpha} G[p] \oplus p^{\alpha} G[p]$ and it follows immediately from the finiteness of the quotient $\left(p^{\alpha} G[p] \oplus p^{\alpha} G[p]\right) /[(H \cap$ $\left.\left.p^{\alpha} G[p]\right) \oplus\left(H \cap p^{\alpha} G[p]\right)\right]$ that $H \cap p^{\alpha} G[p]$ is of finite index in $p^{\alpha} G[p]$, so that $G$ is weakly fully inert socle-regular as claimed.

Conversely, suppose $G$ is a weakly fully inert socle-regular group and let $H$ be an infinite fully inert subgroup of $A=G_{1} \oplus G_{2}$ where $G_{1}=G=G_{2}$. It follows from Lemma 2.1 that $\left(H \cap G_{1}\right) \oplus\left(H \cap G_{2}\right) \sim H$ and each of the $H \cap G_{i}$ is fully inert in $G_{i}$ for $i=1,2$. Two possibilities arise: (i) one of the $H \cap G_{i}$ is finite (note that as $H$ is infinite and commensurable with $\left(H \cap G_{1}\right) \oplus\left(H \cap G_{2}\right)$, it cannot be the case that both of these intersections are finite) or (ii) both of the intersections $H \cap G_{i}$ are infinite.

Consider possibility (i) and assume without loss of generality that $H \cap G_{1}$ is finite. Now let $\phi: G_{2} \rightarrow G_{1}$ be an isomorphism so that $\phi\left(H \cap G_{2}\right)$ is then infinite. However, a further application of Lemma 2.1(ii) yields that the infinite group $\left(H \cap G_{1}\right)+\phi(H \cap$ $\left.G_{2}\right)$ is commensurable with the finite group $H \cap G_{1}$; this contradiction shows that possibility (i) does not arise. 
So we may suppose that both of the intersections $H \cap G_{i}$ are infinite. Since each $G_{i}$ is weakly fully inert socle-regular, there are ordinals $\alpha_{1}, \alpha_{2}$ with $p^{\alpha_{1}} G_{1} \neq 0 \neq p^{\alpha_{2}} G_{2}$ and $\left(\left(H \cap G_{i}\right) \cap p^{\alpha_{i}} G_{i}\right)[p]=H \cap p^{\alpha_{i}} G_{i}[p] \sim p^{\alpha_{i}} G_{i}[p]$ for $i=1,2$. But now an appeal to Proposition 2.3 yields that the desired result: $H \cap p^{\beta} A \sim p^{\beta} A[p]$ where $\beta=\max \left\{\alpha_{1}, \alpha_{2}\right\}$.

Notice that Proposition 3.1 can be strengthened somewhat by restricting our considerations to separable $p$-groups.

Proposition 3.2. If $G$ is a separable and weakly fully inert socle-regular group, then $A=G^{(\kappa)}$ is weakly fully inert socle-regular for any cardinal $\kappa$.

Proof. From Proposition 3.1 we may assume that $\kappa$ is infinite. Furthermore, since we have already established in Corollary 1.5 that direct sums of cyclic groups are even fully inert socle-regular, we may suppose that $G$ is unbounded. Suppose that $H$ is an infinite fully inert subgroup of $A=\bigoplus_{i<\kappa} G_{i}$ where each $G_{i}$ is isomorphic to $G$. Then by Lemma 2.2, the subgroup $H$ is commensurable with $\bigoplus_{i<\kappa} \pi_{i}(H)$, where the $\pi_{i}$ are the canonical projections associated with the decomposition of $A$.

Observe now that since all the $G_{i}$ are isomorphic, it is not possible to have one of $\pi_{i}(H), \pi_{j}(H)$ finite and the other infinite: if $\pi_{i}(H)$ is finite but $\pi_{j}(H)$ is infinite, then $H \cap \pi_{j}(H)$ is of finite index in $\pi_{j}(H)$ and so $H \cap \pi_{j}(H)$ is infinite. If $\phi: G_{j} \rightarrow G_{i}$ is an isomorphism then the quotient $\left(\phi \pi_{j}(H)+H\right) / H \cong \phi \pi_{j}(H) /\left(H \cap \phi \pi_{j}(H)\right)$ is infinite since the numerator of the last term is infinite while the denominator is contained in $\pi_{i}(H)$ which is finite, contrary to the full inertia of $H$.

Assume firstly that all the $\pi_{i}(H)$ are finite; note that infinitely many of the $\pi_{i}(H)$ must be non-zero since $H$ is infinite and commensurable with $\bigoplus_{i<\kappa} \pi_{i}(H)$. Since the $G_{i}$ are unbounded and separable, there exist for each $i<\kappa$, endomorphisms $f_{i}$ of $G_{i}$ with $\pi_{i}(H) \cong f_{i}\left(\pi_{i}(H)\right)$ and $\pi_{i}(H) \cap f_{i}\left(\pi_{i}(H)\right)=0$. Then $f=\bigoplus_{i<\kappa} f_{i}$ is a well-defined endomorphism of $G$ and $(f(H)+H) / H \cong f(H)$ is infinite since it contains the direct sum of infinitely many non-zero subgroups. This contradicts the full inertia of $H$ so we conclude that all the $\pi(H)$ are infinite. 
From Lemma 2.2 we can find a finite subset $I_{0} \subseteq \kappa$ such that $H_{0}=\bigoplus_{i \in \kappa \backslash I_{0}} \pi_{i}(H)$ is fully invariant in $G_{0}=\bigoplus_{i \in \kappa \backslash I_{0}} G_{i}$; as $G_{0}$ is separable it is socle-regular (by Corollary 0.2 in [4]) and so $H_{0}=p^{n_{0}} G_{0}[p]$ for some integer $n_{0}$.

However, for each $i \in I_{0}$, we have that $\pi_{i}(H)$ is fully inert in the weakly fully inert socle-regular $G_{i}$, so we can find integers $n_{i}$ with $\pi_{i}(H) \cap p^{n_{i}} G_{i}[p]$ having finite index in $p^{n_{i}} G_{i}[p]$. Set $u=\max \left\{n_{0}, n_{i}\right\}$ with the $n_{i}$ ranging over the finite set $I_{0}$. Repeated application of Proposition 2.3 gives us that $\bigoplus_{\kappa} \pi_{i}(H) \cap p^{u} G[p] \sim p^{u} G[p]$ and since $H \sim \bigoplus_{\kappa} \pi_{i}(H)$, we have $H \cap p^{u} G[p] \sim p^{u} G[p]$, as required.

We consider now the situation when we form a direct sum of different weakly fully inert socle-regular groups.

We shall also find it convenient to have the following definition:

Definition 3.3. A pair $\left\{G_{1}, G_{2}\right\}$ of reduced $p$-groups with $\ell\left(G_{1}\right)>\ell\left(G_{2}\right)$ is said to be compatible if, for all $\alpha<\ell\left(G_{2}\right)$ there exists a homomorphism $f_{\alpha}: G_{2} \rightarrow G_{1}$ such that $f_{\alpha}\left(p^{\alpha} G_{2}[p]\right)$ is infinite.

Notice that it is necessary for the group $G_{2}$ to be broad if the pair $\left\{G_{1}, G_{2}\right\}$ is to be compatible.

Proposition 3.4. Let $G_{1}, G_{2}$ be reduced p-groups with $\tau_{1}=\ell\left(G_{1}\right)>\ell\left(G_{2}\right)=\tau_{2}$, then if $G_{1}$ is not broad, the group $G=G_{1} \oplus G_{2}$ is not broad and hence $G$ is weakly fully inert socle-regular.

Proof. Let $\alpha<\tau_{1}$ be such that $p^{\alpha} G_{1}$ is finite; note that for all $\beta \geq \alpha$ one has that $p^{\beta} G_{1}$ is finite. Choose $\beta=\max \left\{t_{2}, \alpha\right\}$ and note that $p^{\beta} G=p^{\beta} G_{1}$ is finite with $\beta<\tau_{1}$. Thus $G$ is not broad and the final statement of the proposition follows immediately, as observed in the Introduction.

This simple proposition has some interesting consequences. In particular we have:

Corollary 3.5. (i) If $G$ is a group which is not broad and $\ell(G)>\omega$, then $G \oplus S$ is weakly fully inert socle-regular for any separable group $S$. 
(ii) A direct summand of a weakly fully inert socle-regular group need not be weakly fully inert socle-regular.

Proof. Part (i) is an immediate consequence of the previous proposition and the fact that $\ell(S)=\omega<\ell(G)$.

For part (ii) choose a separable group $S$ which is not weakly fully inert socle-regular - see for example Example 1.6. Now form the direct sum $G=H_{\omega+1} \oplus S$. Then $G$ is weakly fully inert socle-regular but its direct summand $S$ is not.

It can, of course, happen that a summand of a weakly fully inert socle-regular group inherits that property.

Proposition 3.6. Let $G=A \oplus B$ be a weakly fully inert socle-regular group with $\ell(A)=\ell(G)$. Then if every fully inert subgroup of $A$ is commensurable with some fully invariant subgroup of $A$, then $A$ is also weakly fully inert socle-regular.

Proof. Let $H$ be an arbitrary infinite fully inert subgroup of $A$ such that $H \sim M$ for some fully invariant subgroup $M$ of $A$. Let $L$ be the trace of $M$ in $B$, i.e. $L=\Sigma f(M)$ where the summation ranges over all homomorphisms $f \in \operatorname{Hom}(A, B)$; note that $L$ is then fully invariant in $B$. A straightforward calculation shows that $M \oplus L$ is fully invariant in $G$ and so $(M \oplus L) \cap p^{\alpha} G[p] \sim p^{\alpha} G[p]$ for some $\alpha<\ell(G)$ since $G$ is weakly fully inert socle-regular. Now $(M \oplus L) \cap p^{\alpha} G=\left(M \cap p^{\alpha} A\right) \oplus\left(L \cap p^{\alpha} B\right)$, and so $(M \oplus L) \cap p^{\alpha} G[p]=\left(M \cap p^{\alpha} A\right)[p] \oplus\left(L \cap p^{\alpha} B\right)[p] \sim p^{\alpha} A[p] \oplus p^{\alpha} B[p]$. Hence $H \cap p^{\alpha} A[p] \sim M \cap p^{\alpha} A[p] \sim p^{\alpha} A[p]$, as required.

There are two further situations that can arise in our discussion of socle-regularity of groups of the form $G=G_{1} \oplus G_{2}$ where $\ell\left(G_{1}\right)>\ell\left(G_{2}\right)$.

We consider firstly the situation where both $G_{1}, G_{2}$ are broad.

Proposition 3.7. Suppose that $G_{1}, G_{2}$ are broad groups with $\ell\left(G_{2}\right)<\ell\left(G_{1}\right)$. Then if $G=G_{1} \oplus G_{2}$ is weakly fully inert socle-regular, $\left\{G_{1}, G_{2}\right\}$ is a compatible pair.

Proof. : Choose any ordinal $\alpha<\ell\left(G_{2}\right)$ and suppose, for a contradiction, that for every homomorphism $f: G_{2} \rightarrow G_{1}$ one has that $f\left(p^{\alpha} G_{2}[p]\right)$ is finite. Then the 
subgroup $H=0 \oplus p^{\alpha} G_{2}[p]$ is fully inert in $G$ : to see this observe that every endomorphism $\Delta$ of $G$ can be represented as a matrix of the form $\left(\begin{array}{ll}g & h \\ i & j\end{array}\right)$ where $g \in \operatorname{End}\left(G_{1}\right), j \in \operatorname{End}\left(G_{2}\right), i \in \operatorname{Hom}\left(G_{1}, G_{2}\right)$ and $h \in \operatorname{Hom}\left(G_{2}, G_{1}\right)$ and a straightforward check shows that $(H+\Delta(H)) / H \cong h\left(p^{\alpha} G_{2}[p]\right)$ is finite, as required. However, if $\beta$ is any ordinal with $\ell\left(G_{2}\right) \leq \beta<\ell\left(G_{1}\right)$, then $H \cap p^{\beta} G=H \cap$ $p^{\beta} G_{1}=0$, while $p^{\beta} G[p]=p^{\beta} G_{1}[p]$ is infinite since $G_{1}$ is broad. Hence $H \cap p^{\beta} G[p]$ is not commensurable $p^{\beta} G[p]$ for $\ell\left(G_{2}\right) \leq \beta<\ell\left(G_{1}\right)$. Furthermore, $H \cap p^{\lambda} G[p]$ is not commensurable with $p^{\lambda} G[p]$ for any $\lambda<\ell\left(G_{2}\right)$ since this would imply the commensurability of $H \cap p^{\beta} G[p]$ with $p^{\beta} G[p]$ for all $\beta \geq \lambda$. This contradicts the weak full socle-regularity of $G$, so for any $\alpha<\ell\left(G_{2}\right)$ there is a homomorphism $f: G_{2} \rightarrow G_{1}$ with $f\left(p^{\alpha} G_{2}[p]\right)$ infinite, so that $\left\{G_{1}, G_{2}\right\}$ is a compatible pair, as required.

Our next result is a partial converse to Proposition 3.7 above.

Proposition 3.8. Let $G_{1}, G_{2}$ be broad, weakly fully inert socle-regular groups with $\ell\left(G_{2}\right)<\ell\left(G_{1}\right)$ such that $\left\{G_{1}, G_{2}\right\}$ is a compatible pair, then $G=G_{1} \oplus G_{2}$ is also weakly fully inert socle-regular.

Proof. Let $H \leq G[p]$ be an infinite fully inert subgroup of $G$. Then we know that $H \sim\left(H \cap G_{1}\right) \oplus\left(H \cap G_{2}\right)$ and that for $i=1,2$ we have that $H \cap G_{i}$ is fully inert in $G_{i}$.

There are three cases to be considered:

(i) $\left(H \cap G_{1}\right),\left(H \cap G_{2}\right)$ are both infinite; (ii) $H \cap G_{2}$ is finite; (iii) $H \cap G_{1}$ is finite. Note that both $H \cap G_{1}$ and $H \cap G_{2}$ cannot be finite since $H$ is infinite and $H \sim$ $\left(H \cap G_{1}\right) \oplus\left(H \cap G_{2}\right)$.

Case(i). In this case the weak full inert socle-regularity of $G$ follows directly from an application of Proposition 2.3.

Case(ii). Suppose that $H \cap G_{2}$ is finite. Then $H \sim\left(H \cap G_{1}\right) \oplus\left(H \cap G_{2}\right) \sim H \cap G_{1}$, so that $H \cap G_{1}$ is infinite and fully inert in $G_{1}$. Since $G_{1}$ is weakly fully inert socle-regular, there is an ordinal $\beta<\ell\left(G_{1}\right)$ such that

$$
\left(H \cap G_{1}\right) \cap p^{\beta} G_{1}[p]=H \cap p^{\beta} G_{1}[p] \sim p^{\beta} G_{1}[p] .
$$


Now let $\delta=\max \left\{\beta, \ell\left(G_{2}\right)\right\}$; note that $\delta<\ell\left(G_{1}\right)=\ell(G)$. Since $\delta \geq \beta$ we have by the usual argument that $H \cap p^{\delta} G_{1}[p] \sim p^{\delta} G_{1}[p]$ and this last term is equal to $p^{\delta} G[p]$ since $p^{\delta} G_{2}=0$. However, for the same reason one also has that $H \cap p^{\delta} G \sim H \cap p^{\delta} G_{1}$ and so $H \cap p^{\delta} G \sim H \cap p^{\delta} G_{1} \sim p^{\delta} G[p]$.

Case(iii). Suppose that $H \cap G_{1}$ is finite. We show that this case cannot arise, so suppose, for a contradiction, that $H \cap G_{1}$ is finite. Now $H \sim\left(H \cap G_{1}\right) \oplus\left(H \cap G_{2}\right)$ and since $H$ is infinite we conclude that $H \cap G_{2}$ is also infinite; it is also fully inert in $G_{2}$, so we have that there is an ordinal $\gamma<\ell\left(G_{2}\right)$ with $H \cap p^{\gamma} G_{2} \sim p^{\gamma} G_{2}[p]$. The finiteness of $H \cap G_{1}$ also gives that $H \sim H \cap G_{2}$ and hence the latter is also fully inert in $G$. Furthermore, since we have by assumption that $\left\{G_{1}, G_{2}\right\}$ is a compatible pair, there is an homomorphism $f_{\gamma}: G_{2} \rightarrow G_{1}$ with $f_{\gamma}\left(p^{\gamma} G_{2}[p]\right)$ infinite. Note that since $H \cap p^{\gamma} G_{2} \sim p^{\gamma} G_{2}[p]$, we also have that $f_{\gamma}\left(H \cap p^{\gamma} G_{2}\right)$ is infinite.

Now consider the mapping $\Delta=\left(\begin{array}{cc}0 & f_{\gamma} \\ 0 & 0\end{array}\right)$ which represents an endomorphism of $G$. Apply this choice of $\Delta$ to calculate the quotient $\left[\left(H \cap G_{2}\right)+\Delta\left(H \cap G_{2}\right)\right] /\left(H \cap G_{2}\right)$, noting that the quotient must be finite since $H \cap G_{2}$ is fully inert in $G$. This yields that $\left(\left(H \cap G_{2}\right)+f_{\gamma}\left(H \cap G_{2}\right)\right) /\left(H \cap G_{2}\right) \cong f_{\gamma}\left(H \cap G_{2}\right)$ is finite. This is a contradiction since $f_{\gamma}\left(H \cap G_{2}\right)$ contains the infinite group $f_{\gamma}\left(H \cap p^{\gamma} G_{2}\right)$. Hence Case (iii) cannot occur.

Combining the last two propositions we obtain

Theorem 3.9. Let $G_{1}, G_{2}$ be broad, inertly socle-regular groups with $\ell\left(G_{2}\right)<\ell\left(G_{1}\right)$, then the direct sum $G=G_{1} \oplus G_{2}$ is weakly fully inert socle-regular if, and only if, $\left\{G_{1}, G_{2}\right\}$ is a compatible pair.

We now consider the situation in which the groups $G_{1}, G_{2}$ are both of length $\tau$. It seems useful to modify the definition of compatible as follows:

Definition 3.10. A pair $\left\{G_{1}, G_{2}\right\}$ of reduced $p$-groups with $\ell\left(G_{1}\right)=\ell\left(G_{2}\right)=\tau$ is said to be fully compatible if, for all $\alpha<\tau$ there exist homomorphisms $f_{\alpha}: G_{2} \rightarrow G_{1}$ and $f_{\alpha}^{\prime}: G_{1} \rightarrow G_{2}$ such that both $f_{\alpha}\left(p^{\alpha} G_{2}[p]\right)$ and $f_{\alpha}^{\prime}\left(p^{\alpha} G_{1}[p]\right)$ are infinite.

We have the following result corresponding to Proposition 3.8: 
Proposition 3.11. Let $G_{1}, G_{2}$ be broad, weakly fully inert socle-regular groups with $\ell\left(G_{2}\right)=\ell\left(G_{1}\right)=\tau$ such that $\left\{G_{1}, G_{2}\right\}$ is a fully compatible pair, then $G=G_{1} \oplus G_{2}$ is also weakly fully inert socle-regular.

Proof. Let $H \leq G[p]$ be an infinite fully inert subgroup of $G$. Then we know that $H \sim\left(H \cap G_{1}\right) \oplus\left(H \cap G_{2}\right)$ and that for $i=1,2$ we have that $H \cap G_{i}$ is fully inert in $G_{i}$.

Exactly as in Proposition 3.8 there are three cases to be considered:(i) $\left(H \cap G_{1}\right),(H \cap$ $\left.G_{2}\right)$ are both infinite; (ii) $\left(H \cap G_{2}\right)$ is finite; (iii) $\left.H \cap G_{1}\right)$ is finite.

Note that both $H \cap G_{1}$ and $H \cap G_{2}$ cannot be finite since $H$ is infinite and $H \sim$ $\left(H \cap G_{1}\right) \oplus\left(H \cap G_{2}\right)$.

Case (i) follows exactly as in Proposition 3.8 above.

Case (ii) Suppose that $H \cap G_{2}$ is finite. Then $H \sim\left(H \cap G_{1}\right) \oplus\left(H \cap G_{2}\right)$ implies that $H \cap G_{1}$ is infinite. Since $\left(H \cap G_{1}\right)$ is fully inert in the socle-regular group $G_{1}$, we can find $\beta<\tau$ with $H \cap p^{\beta} G_{1} \sim p^{\beta} G_{1}[p]$.

Now consider any $\phi: G_{1} \rightarrow G_{2}$; we know from Lemma 2.2 that $\left(H \cap G_{2}\right)+\phi(H \cap$ $\left.G_{1}\right) \sim H \cap G_{2}$. So $\left(\left(H \cap G_{2}\right)+\phi\left(H \cap G_{1}\right)\right) /\left(H \cap G_{2}\right)$ is finite. Applying the first isomorphism theorem and noting that the intersection $\left(H \cap G_{2}\right) \cap \phi\left(H \cap G_{1}\right)$ is finite by assumption in this case, we deduce that $\phi\left(H \cap G_{1}\right)$ is finite for all $f: G_{1} \rightarrow G_{2}$. This is impossible: $\phi\left(H \cap G_{1}\right) \geq \phi\left(H \cap p^{\beta} G_{1}\right) \sim \phi\left(p^{\beta} G_{2}[p]\right)$, giving that $\phi\left(p^{\beta} G_{1}[p]\right)$ is finite, contrary to $\left\{G_{1}, G_{2}\right\}$ being fully compatible.

Case (iii) Suppose that $H \cap G_{1}$ ) is finite. This is now an identical argument to Case (ii), utilising the symmetry inherent in the definition of full compatibility.

\section{Subgroups of (WeAkly) Fully inert Socle-REgular Groups}

In this section we consider how the properties of being weakly fully inert or fully inert socle-regular are reflected in subgroups of the group.

Our first result is the interesting but straightforward:

Proposition 4.1. If $G$ is a weakly fully inert socle-regular group, then for any ordinal $\alpha$, if $p^{\alpha} G$ is infinite, $p^{\alpha} G$ is also weakly fully inert socle-regular. 
Proof. Let $H$ be an infinite fully inert subgroup of $p^{\alpha} G$. Since $p^{\alpha} G$ is fully invariant in $G, H$ is also fully inert in $G$. Thus there is an ordinal $\sigma$ with $p^{\sigma} G \neq 0$ such that $\left(H \cap p^{\sigma} G\right) \sim p^{\sigma} G[p]$. But then $\left(H \cap p^{\sigma} G\right)[p] \cap p^{\alpha} G \sim p^{\sigma} G[p] \cap p^{\alpha} G$ so that $\left(H \cap p^{\beta} G\right)[p] \sim p^{\beta} G[p]$ where $\beta=\max \{\alpha, \sigma\}$. Note that $p^{\beta} G \neq 0$ since $p^{\sigma} G \neq 0$ and $p^{\alpha} G$ is infinite. Writing $\beta=\alpha+\lambda$, for some $\lambda$, we have that $\left(H \cap p^{\lambda}\left(p^{\alpha} G\right)\right)[p] \sim$ $\left(p^{\lambda}\left(p^{\alpha} G\right)\right)[p]$ and $p^{\alpha} G$ is weakly fully iner socle-regular, as required.

Next we wish to investigate the relationship between a fully inert socle-regular group and its finite index subgroups.

We need two technical results:

Lemma 4.2. If $A=B \oplus F$ is a group with a finite subgroup $F$, then $Y \oplus F$ is fully inert in A provided $Y$ is fully inert in $B$.

Proof. An arbitrary endomorphism of the group $A$ has the form $\Delta=\left(\begin{array}{ll}\alpha & \gamma \\ \delta & \beta\end{array}\right)$, where $\alpha: B \rightarrow B, \gamma: F \rightarrow B, \delta: B \rightarrow F$ and $\beta: F \rightarrow F$ are homomorphisms. Then, one sees that $\Delta(Y \oplus F)=(\alpha(Y)+\gamma(F)) \oplus(\delta(Y)+\beta(F))$, so that

$$
\frac{\Delta(Y \oplus F)+Y \oplus F}{Y \oplus F}=\frac{[Y+\alpha(Y)+\gamma(F)] \oplus F}{Y \oplus F} \cong \frac{Y+\alpha(Y)+\gamma(F)}{Y}
$$

Set $Z=Y+\alpha(Y)$ to get that $\frac{\Delta(Y \oplus F)+Y \oplus F}{Y \oplus F} \cong \frac{Z+\gamma(F)}{Y}$. Now, as $Y$ is fully inert in $B$, one concludes that $\frac{Z}{Y}=\frac{Y+\alpha(Y)}{Y} \cong \frac{\alpha(Y)}{Y \cap \alpha(Y)}$ is necessarily finite. Furthermore,

$$
\frac{Z+\gamma(F)}{Y} / \frac{Z}{Y} \cong \frac{Z+\gamma(F)}{Z} \cong \frac{\gamma(F)}{Z \cap \gamma(F)}
$$

is finite. Since $\frac{Z}{Y}$ is also finite, we have that $\frac{Z+\gamma(F)}{Y}$ must be finite, and so $Y \oplus F$ is fully inert in $A$, as required.

As an immediate consequence, we obtain:

Corollary 4.3. If $A=B \oplus F$ is fully inert socle-regular group, where $F \leq A$ is a finite subgroup, then $B$ is fully inert socle-regular. 
Proof. If $Y$ is a fully inert subgroup in $B$, then a direct check in Lemma 4.2 shows that the direct sum $Y \oplus F$ is fully inert in $A$. Thus, $(Y \oplus F)[p] \sim\left(p^{\delta} A\right)[p]$ for some ordinal $\delta \geq 0$ and so $Y[p] \oplus F[p] \sim\left(p^{\delta} A\right)[p]=\left(p^{\delta} B\right)[p] \oplus\left(p^{\delta} F\right)[p]$. But then $Y[p] \sim\left(p^{\delta} A\right)[p] \sim\left(p^{\delta} B\right)[p]$ gives the required $Y[p] \sim\left(p^{\delta} B\right)[p]$ by the transitivity of the commensurability relation.

It is now easy to obtain the connection we desired:

Theorem 4.4. If $G$ is a group with a finite index subgroup $A$, then $G$ is fully inert socle-regular if, and only if, A is fully inert socle-regular.

Proof. First of all, we need the following well-known general observation which may be found in, for example, [13, Lemma 16.5]: if $A$ is of finite index in $G$, we can find a direct summand $C$ of $G$ with $C \leq A$ such that the quotient $G / C$ is finite. Thus, we have the two decompositions $G=C \oplus F$ and $A=C \oplus(A \cap F)$ with $F$ finite. To prove necessity, assume $G$ is fully inert socle-regular and suppose $H$ is an arbitrary infinite fully inert subgroup of $A$. Then, $H \cap C$ is infinite and fully inert in $C$ and $H /(H \cap C) \cong(H+C) / C \leq A / C \leq G / C$ is finite. So, $H \cap C \sim H$ implying that $(H \cap C)[p] \sim H[p]$. Utilizing now Corollary 4.3, one sees that $C$ is fully inert socle-regular. Consequently, $(H \cap C)[p] \sim\left(p^{\beta} C\right)[p]$ for some ordinal $\beta$. But using the symmetry of the operation $\sim$, it follows that $H[p] \sim(H \cap C)[p]$ and hence, by the transitivity property of the same operation, we obtain that $H[p] \sim\left(p^{\beta} C\right)[p]$. However, $p^{\beta} A=p^{\beta} C \oplus p^{\beta}(A \cap F)$ whence $\left(p^{\beta} A\right)[p] \sim\left(p^{\beta} C\right)[p]$ and so, again by symmetry, $H[p] \sim\left(p^{\beta} C\right)[p] \sim\left(p^{\beta} A\right)[p]$ giving us again by transitivity that $H[p] \sim\left(p^{\beta} A\right)[p]$, as required.

To establish sufficiency, assume $A$ is fully inert socle-regular and suppose $X$ is an arbitrary fully inert subgroup of $G$. Then Corollary 4.3 above again tells us that $C$ is fully inert socle-regular. Now an easy check shows that $X \cap C$ is fully inert in $C$, whence $(X \cap C)[p] \sim\left(p^{\gamma} C\right)[p]$ for some $\gamma \geq 0$. But $X /(X \cap C) \cong(X+C) / C \leq G / C$ is finite, yielding that $X \cap C \sim X$ and hence that $(X \cap C)[p] \sim X[p]$. Therefore, we deduce as above that $X[p] \sim(X \cap C)[p] \sim\left(p^{\gamma} C\right)[p]$ forcing $X[p] \sim\left(p^{\gamma} C\right)[p]$. But 
$p^{\gamma} G \sim p^{\gamma} C$ ensures that $\left(p^{\gamma} G\right)[p] \sim\left(p^{\gamma} C\right)[p]$ and so, finally, $X[p] \sim\left(p^{\gamma} G\right)[p]$, as required.

There is another situation in which we can characterize weak fully inert socleregularity in terms of subgroups. First we make a simple observation which is useful in simplifying our arguments.

Lemma 4.5. Suppose that $H \leq G[p]$ is an infinite fully inert subgroup of $G$ and $N$ is an arbitrary infinite subgroup of $G$. Then a sufficient condition to ensure that the subgroup $H \cap N$ be infinite is:

- there exists an endomorphism $\phi$ of $G$ with $\phi(H)$ infinite and $\phi(H) \leq N$.

Proof. The proof is straightforward: since $H$ is fully inert in $G$, the quotient $(H+$ $\phi(H)) / H \cong \phi(H) /(H \cap \phi(H))$ is finite. By assumption $\phi(H)$ is infinite, so $H \cap \phi(H)$ must also be infinite. However, $H \cap \phi(H) \leq H \cap N$, so the latter must also be infinite, as required.

So, we come to our basic tool in this section.

Theorem 4.6. Suppose that $G$ is an infinite reduced $p$-group such that $G / p^{\omega} G$ is a non-trivial direct sum of cyclic groups. Then $G$ is weakly fully inert socle-regular if, and only if, $p^{\omega} G$ is either weakly fully inert socle-regular or finite.

Proof. It follows from Proposition 4.1 that if $G$ is an arbitrary weakly fully inert socle-regular group, then either $p^{\alpha} G$ is finite or it also weakly fully inert socle-regular for any ordinal $\alpha$.

Conversely, suppose that $p^{\omega} G$ is finite so that $G$ is not broad and it follows that $G$ is weakly fully inert socle-regular. So suppose that $p^{\omega} G$ is infinite weakly fully inert socle-regular and let $H$ be an arbitrary infinite fully inert subgroup of $G$ with $H \leq G[p]$; note that $G$ is unbounded in this situation and so $G / p^{\omega} G$ is also unbounded by a theorem of Kulikov - see, for example, Theorem 30.2 [7]. Since $G / p^{\omega} G$ is a direct sum of cyclic groups we have, by an easy extension of Zippin's Theorem, that every endomorphism of $p^{\omega} G$ extends to an endomorphism of $G$ and it follows from Lemma 2.1 that $H \cap p^{\omega} G$ is fully inert in $p^{\omega} G$. Now consider the 
intersection $H \cap p^{\omega} G$; there are two possibilities, the intersection is either finite or infinite.

We show that the first possibility cannot arise making use of Lemma 4.5 above: we will exhibit an endomorphism $\phi$ of $G$ with $\phi(H)$ infinite and $\phi(H) \leq p^{\omega} G$.

Let $\pi: G \rightarrow \bar{G}=G / p^{\omega} G$ be the canonical projection, then $\bar{H}=\pi(H) \cong H /(H \cap$ $\left.p^{\omega} G\right)$ is an infinite subgroup of $\bar{G}[p]$. Choose a countably infinite subgroup $\bar{L}$ of $\bar{H}$. Since $\bar{G}$ is a direct sum of cyclic groups, a standard support argument yields a direct summand, $\bar{K}$ say, of $\bar{G}$ with $\bar{L} \leq \bar{K}[p]$; let $\bar{K}=\bigoplus_{i=1}^{\infty}\left\langle\bar{k}_{i}\right\rangle$, where $o\left(\bar{k}_{i}\right)=p^{n_{i}}$.

Now choose a countably infinite subgroup $S$ of $p^{\omega} G[p]$ - this is possible since $p^{\omega} G$ is infinite. Let $S=\bigoplus_{i=1}^{\infty}\left\langle s_{i}\right\rangle$; as $s_{i} \in p^{\omega} G$ we can find elements $x_{i} \in G$ with $s_{i}=p^{n_{i}-1} x_{i}$. Define a function $f: \bar{G} \rightarrow G$ by setting $f\left(\bar{k}_{i}\right)=x_{i}$ and setting $f$ to be identically zero on a direct complement of $\bar{K}$. This gives us a well-defined homomorphism $\bar{G} \rightarrow G$ since $o\left(x_{i}\right)=p^{n_{i}}=o\left(\bar{k}_{i}\right)$. Since the elements $s_{i}$ are independent, an easy argument gives us that $f \uparrow \bar{K}[p]$ is monic and hence $f(\bar{L}) \cong \bar{L}$ is infinite.

Now, define $\phi: G \rightarrow G$ as the composition $\phi=f \pi$ and note that $\phi(H)=f(\bar{H}) \geq$ $f(\bar{L})$ is infinite. Also $\phi(H)=f(\bar{H}) \leq f(\bar{G}[p])=f(\bar{K}[p])=S \leq p^{\omega} G$. Hence $\phi$ is an endomorphism of $G$ with $\phi(H)$ infinite and $\phi(H) \leq p^{\omega} G$, so $H \cap p^{\omega} G$ is necessarily infinite.

So we have that $H \cap p^{\omega} G$ is an infinite fully inert subgroup of the weakly fully inert socle-regular group $p^{\omega} G$. Hence there is an ordinal $\alpha$ with $p^{\alpha}\left(p^{\omega} G\right)=p^{\omega+\alpha} G \neq 0$ such that $\left(H \cap p^{\omega} G\right) \cap p^{\alpha}\left(p^{\omega} G\right) \sim p^{\alpha}\left(p^{\omega} G\right)[p]$. Setting $\beta=\omega+\alpha$, we have that $H \cap p^{\beta} G \sim p^{\beta} G[p]$ and $p^{\beta} G \neq 0$. Since $H$ was arbitrary, we conclude that $G$ is weakly fully inert socle-regular.

The next consequence is immediate.

Corollary 4.7. Any totally projective group $G$ (in particular any countable group) of length not exceeding $\omega \cdot 2$ is weakly fully inert socle-regular.

Proof. The totally projective group $G / p^{\omega} G$ is then separable and hence is a direct sum of cyclic groups. The Ulm subgroup $p^{\omega} G$ is also totally projective and it is separable by the restriction on the length of $G$, so it too is a direct sum of cyclic groups 
and hence, by Corollary 1.5 it is weakly fully inert socle-regular. The corollary now follows immediately from the previous theorem.

The following example shows that the conditions on the first Ulm subgroup and the first Ulm factor in Theorem 4.6 above cannot be omitted. In fact, we shall construct an uncountable group $G$ which is not weakly fully inert socle-regular with a countable first Ulm subgroup $p^{\omega} G$ such that the first Ulm factor $G / p^{\omega} G$ is not a direct sum of cyclic groups. Our example is closely related to that given in Examples 1.6 and 1.7 and utilizes again the standard $p$-group $P$ first constructed by Pierce.

Example 4.8. There exists a reduced group $G$ with $p^{\omega} G$ elementary of countably infinite rank and a fully inert subgroup $H$ of $G$ such that $\left(H \cap p^{\alpha} G\right)[p]$ is not of finite index in $\left(p^{\alpha} G\right)[p]$ for any $\alpha$ with $0 \leq \alpha<\omega+1$. In particular, $G$ is an uncountable group which is not weakly fully inert socle-regular group and the Ulm factor $G / p^{\omega} G$ is not a direct sum of cyclic groups.

Proof. The proof of the example is based around the construction of a group $G$ with $p^{\omega} G=V=\bigoplus_{\aleph_{0}} \mathbb{Z}(p)$ and $G / p^{\omega} G \cong P$. Let $C=C_{1} \oplus \cdots \oplus C_{n} \oplus \ldots$ be a basic subgroup of such a group $G$ and note that it follows from a well-known result of Kulikov that $C \cong B$ (see, e.g., [7, Theorem 30.2]).

Assume for the moment that we have constructed such a group and take $H=C[p]$; we claim that $H$ is then fully inert in $G$. Now, if $\phi \in \operatorname{End}(G)$, then $\phi=r 1_{G}+\theta$ for some $p$-adic integer $r$ and $\theta \in E_{s}(G)$. Thus $H+\phi(H)=H+\theta(H)$. Since $\theta$ is small, it is easy to see that $\theta(H)=\theta\left(C_{1} \oplus \cdots \oplus C_{N}\right)$ for some integer $N$ - see the argument in [11, Lemma 4.1] observing that separability is not required for that lemma. Hence $(H+\phi(H)) / H$ is a homomorphic image of $\theta(H)$ which is finite since $C$ is standard. So $H=C[p]$ is certainly fully inert in $G$.

To show that $\left(H \cap p^{\alpha} G\right)[p]$ is not of finite index in $p^{\alpha} G[p]$ for any $\alpha<\omega+1$, we deal firstly with the case where $\alpha$ is an integer $n$. Then, since $G / p^{n} G \cong C / p^{n} C$ is countable while $G$ is uncountable, we conclude that $\left|p^{n} G\right|=2^{\aleph_{0}}$ and so $\left|p^{n} G[p]\right|=$ $2^{\aleph_{0}}$ also. However, $\left|\left(C \cap p^{n} G\right)[p]\right| \leq|C|=\aleph_{0}$, so the index of $\left(C \cap p^{n} G\right)[p]$ in $p^{n} G[p]$ 
is actually uncountable. Finally, note that $C[p] \cap p^{\omega} G=\{0\}$ since $C$ is basic in $G$ and thus $p^{\omega} G[p] /\left(C[p] \cap p^{\omega} G\right)[p] \cong p^{\omega} G[p] \cong V$ is infinite.

Thus the construction of our example will be complete once we have carried out the construction of the group $G$ - note that the argument for this is by now fairly standard and utilizes a variant of an argument used in [14, Lemma 4.4].

Define the groups $G_{0}=P, G_{1}=V$ as above. Then it is straightforward to check that the $G_{\alpha}(\alpha=0,1)$ satisfy the conditions of Kulikov's Theorem [9, Theorem 10.1.9] and so there is a group $G$ with $p^{\omega} G=V$ and $G / p^{\omega} G \cong P$. Now consider an arbitrary endomorphism $\phi \in \operatorname{End}(G)$. This induces an endomorphism of $G / p^{\omega} G$ which is of the form $r 1_{G / p^{\omega} G}+\chi$, where $\chi$ is a small endomorphism of $G / p^{\omega} G$. Then $\theta=\phi-r 1_{G}$ induces $\chi$ on $G / p^{\omega} G$, and so it follows from [3, Lemma 7.1(ii)] that $\theta$ is thin. However, as $G$ has length $\omega+1<\omega+\omega$, we actually have that $\theta$ is a small endomorphism of $G$ - see [3, Corollary 7.7]. So, $\operatorname{End}(G) \leq J_{p} \cdot 1_{G} \oplus E_{s}(G)$ and since the reverse inequality always holds, we have $\operatorname{End}(G)=J_{p} \cdot 1_{G} \oplus E_{s}(G)$, as required.

Proposition 4.9. If $H \leq G[p]$ is fully inert in $G$ and $H \cap p^{n} G \sim p^{n} G[p]$ for some non-negative integer $n$, then there exists a non-negative integer $m$, with $m \leq n$ such that $H \sim p^{m} G[p]$.

Proof. If $n=0$, then $H=H \cap p^{n} G \sim p^{n} G[p]$ and we are finished by choosing $m=0$. So we may suppose that $n \geq 1$. Choose $m$ to be the least integer such that $H \cap p^{m} G \sim p^{m} G[p]$ - such an $m$ exists by an earlier observation and we may assume from the previous argument that $m \geq 1$. Then $H \cap p^{m-1} G[p]$ is not commensurable with $p^{m-1} G[p]$. Now suppose, for a contradiction, that $H$ is not commensurable with $p^{m} G[p]$.

Since $H \cap p^{m} G[p] \sim p^{m} G[p]$, we must then have that $H /\left(H \cap p^{m} G[p]\right)$ is infinite. Now $H \cap p^{m-1} G$ does not have finite index in $p^{m-1} G[p]=B_{m}[p] \oplus p^{m} G[p]$, but as every endomorphism of $p^{m-1} G$ extends to an endomorphism of $G$ (this is an easy extension of Proposition 113.3 in [8] - details are given in [6, Lemma 2.11]) it then follows from Lemma 2.1(i) that $H \cap p^{m-1} G$ is fully inert in $p^{m-1} G$. Appealing 
to Lemma 2.1 (ii), we conclude that $H \cap p^{m-1} G$ is fully inert in $B_{m}[p]$ and that $\left(\left(H \cap p^{m-1} G\right) \cap B_{m}[p]\right) \oplus\left(H \cap p^{m-1} G \cap p^{m} G[p]\right)$ is of finite index in $H \cap p^{m-1} G$. Since $H \cap p^{m-1} G$ is not of finite index in $p^{m-1} G[p]$, we conclude, doing some simple tidying up of terms, that $\left(B_{m}[p] /\left(H \cap B_{m}[p]\right)\right) \oplus\left(p^{m} G[p] /\left(H \cap p^{m} G[p]\right)\right)$ is infinite. Since the last term in this expression is finite, we must have that $B_{m}[p] /\left(H \cap B_{m}[p]\right)$ is infinite.

However, as $B_{m}$ is a summand of $G$, we also have that $H \cap B_{m}$ is fully inert in the homocyclic group $B_{m}$. It follows from Lemma 2.4 that either $H \cap B_{m}$ is finite or $H \cap B_{m}=H \cap B_{m}[p]$ is cofinite in $B_{m}[p]$. Since the second possibility contradicts the fact that $B_{m}[p] /\left(H \cap B_{m}[p]\right)$ is infinite, we must have that $H \cap B_{m}[p]$ is finite. As $B_{m}[p]$ is finite, there must exist a $k<m$ such that $H \cap B_{k}[p]$ is infinite; otherwise $H \cap B_{k}[p]$ is finite for all $k \leq m$ and as $G=B_{1} \oplus \cdots \oplus B_{m} \oplus G_{m}^{*}$, we have that $H$ is commensurable with $\left(H \cap B_{1}\right) \oplus \cdots \oplus\left(H \cap B_{m}\right) \oplus\left(H \cap G_{m}^{*}\right)$, so that $H \sim H \cap G_{m}^{*}=$ $H \cap G_{m}^{*}[p]$. But it is well known (and easy to prove) that $G_{m}^{*}[p]=p^{m} G[p]$ so we have $H \sim H \cap p^{m} G[p]$ - contrary to our hypothesis.

This leads immediately to a conflict with Lemma 2.5: if $H \cap B_{k}[p]$ is infinite and $k<m$, then $H \cap B_{m}[p]$ is also infinite. This final contradiction establishes the proposition.

We can now derive the following curious consequence.

Corollary 4.10. If $G$ is a separable weakly fully inert socle-regular group, then $G$ is fully inert socle-regular.

\section{The Restrictive Case}

As promised in the Introduction, we now show that the definition requiring the existence of an ordinal $\alpha$ with $H[p]=p^{\alpha} G[p]$ is rather too restrictive.

First we have a simple technical lemma; recall that $f_{n}(G)$ denotes $n$th Ulm-Kaplansky invariant of the group $G$, where $n$ is a non-negative integer.

Lemma 5.1. If $G$ is a reduced group and $f_{n}(G) \geq 2$ for some integer $n \geq 0$, then there is a fully inert subgroup $H$ of $G$ such that $H[p] \neq p^{\alpha} G[p]$ for any ordinal $\alpha \geq 0$. 
Proof. Since the Ulm-Kalpansky of $G$ are greater than or equal to 2, there are elements $x, y$ in $G$ such that $o(x)=p^{n+1}=o(y)$ and such that $G=\langle x\rangle \oplus\langle y\rangle \oplus G^{\prime}$ for some $G^{\prime} \leq G$. Let $H=\langle y\rangle \oplus G^{\prime}$, so $H$ is of finite index in $G$ and hence $H$ is fully inert in $G$. Claim that $H[p] \neq\left(p^{\alpha} G\right)[p]$ for any ordinal $\alpha \geq 0$. Set $e=p^{n} x, f=p^{n} y$ and note that $e, f$ are elements of order $p$ having height exactly $n$ as computed in $G$. It follows that $G[p]=\langle e\rangle \oplus\langle f\rangle \oplus G^{\prime}[p]$ and $H[p]=\langle f\rangle \oplus G^{\prime}[p]$. We now consider the three possibilities that arise for an arbitrary fixed ordinal $\alpha$ :

- If $\alpha=0$, then $e \in G[p] \backslash H[p]$;

- If $\alpha \geq n+1$, then $f \in H[p]$ but $f \notin\left(p^{\alpha} G\right)[p]$ since, as noted above, $f$ has height precisely $n$ in $G$;

- If $1 \leq \alpha \leq n$, then $p^{\alpha} G=p^{\alpha}\langle x\rangle \oplus p^{\alpha} H$ and so $e \in\left(p^{\alpha} G\right)[p] \backslash H[p]$.

Therefore, $H[p] \neq\left(p^{\alpha} G\right)[p]$ for any $\alpha \geq 0$ as required.

Lemma 5.2. Suppose $G$ is a reduced group and $n \geq 0$ is the smallest integer such that $f_{n}(G) \neq 0$. If there exists an integer $m>n$ with $f_{m}(G) \neq 0$, then there is a fully inert subgroup $H$ of $G$ such that $H[p] \neq p^{\alpha} G[p]$ for any ordinal $\alpha \geq 0$.

Proof. Under the hypotheses above, there is a decomposition $G=\langle g\rangle \oplus\langle h\rangle \oplus K$ for some $K \leq G$ and elements $g, h \in G$ with $o(g)=p^{n+1}<o(h)=p^{m+1}$. Setting $H=\langle g\rangle \oplus K$, one sees that $H$ is a finite index subgroup of $G$, whence it is fully inert subgroup in $G$. Now $H[p]=\left\langle p^{n} g\right\rangle \oplus K[p]$ and we distinguish two possible cases:

- If $\alpha \geq n+1$, then $p^{\alpha} G=p^{\alpha}\langle h\rangle \oplus p^{\alpha} K$ and so $0 \neq p^{n} g \in H[p]$ but clearly $p^{n} g \notin\left(p^{\alpha} G\right)[p]$.

- If $\alpha \leq n$, then $\alpha<m$ and $0 \neq p^{m} h \in\left(p^{\alpha} G\right)[p]$ but clearly $p^{m} h \notin H[p]$.

Thus the inequality $H[p] \neq\left(p^{\alpha} G\right)[p]$ holds for any $\alpha \geq 0$, as required.

We are ready to classify those groups satisfying the restrictive hypothesis:

(RH) For all fully inert subgroups $H$ of $G$ there exists an ordinal $\alpha$, depending on $H$, such that $H[p]=p^{\alpha} G[p]$.

Theorem 5.3. A reduced group satisfies the hypothesis $(\mathrm{RH})$ if, and only if, it is cyclic. 
Proof. The sufficiency is pretty straightforward. In fact, if $G$ is cyclic, then the fully inert subgroups of $G$ are just the set of all subgroups of $G$ and thus if $H \leq G$, then $H[p]=G[p]$, as expected.

Conversely, it follows at once from Lemma 5.2 that the group $G$ has at most one non-zero finite Ulm-Kaplansky invariant $f_{n}(G)$. Furthermore, Lemma 5.1 tells us that $f_{n}(G)$ is at most 1 . Hence a basic subgroup of $G$, and thus $G$ itself, is cyclic, as required.

\section{Open Problems}

We conclude our discussions with three still left-open problems, the solution of which may lead to further insight into the classes of (weakly) fully inert socle-regular groups.

Problem 1. Is it true that a group $G$ is fully inert socle-regular if, and only if, the direct sum $G \oplus G$ is fully inert socle-regular?

Problem 2. Is it true that a totally projective group is (weakly) fully inert socleregular?

Problem 3. Does Theorem 4.6 remain true, provided that the quotient-group $G / p^{\alpha} G$ is non-trivial totally projective for some ordinal $\alpha$ ?

Acknowledgements. The authors would like to express their sincere thanks to the anonymous expert referee for the competent comments and suggestions made as well as thanks are due to the handling editor, Professor Francesco de Giovanni, for his professional management of the article.

Funding: The work of P.V. Danchev is partially supported by the Bulgarian National Science Fund under Grant KP-06 No 32/1 of December 07, 2019. 


\section{REFERENCES}

[1] A.R. Chekhlov, Fully inert subgroups of completely decomposable finite rank groups and their commensurability, Vestn. Tomsk. Gos. Univ. Math. \& Mech. (3) 41 (2016), 42-50.

[2] A.R. Chekhlov, On fully inert subgroups of completely decomposable groups, Math. Notes (2) 101 (2017), 365-373.

[3] A.L.S. Corner, On endomorphism rings of primary Abelian groups II, Quart. J. Math. Oxford 27 (1976), 5-13.

[4] P. Danchev and B. Goldsmith, On the socles of fully invariant subgroups of Abelian p-groups, Arch. Math. (3) 92 (2009), 191-199.

[5] P. Danchev and B. Goldsmith, On the socles of characteristic subgroups of Abelian p-groups, J. Algebra 323 (2010), 3020-3028.

[6] P. Danchev and B. Goldsmith, On commutator socle-regular Abelian p-groups, J. Group Theory 17 (2014) 781-803.

[7] L. Fuchs, Abelian Groups, Publ. House of the Hungar. Acad. Sci., Budapest, 1958.

[8] L. Fuchs, Infinite Abelian Groups, Vol. I \& II, Acad. Press, New York and London, 1970 \& 1973.

[9] L. Fuchs, Abelian Groups, Springer, Switzerland (2015).

[10] B. Goldsmith, Essentially-rigid families of Abelian p-groups, J. London Math. Soc. (2) 18 (1978) 70-74.

[11] B. Goldsmith, L. Salce and P. Zanardo, Fully inert subgroups of Abelian p-groups, J. Algebra 419 (2014), 332-349.

[12] B. Goldsmith and L. Salce, Fully inert subgroups of torsion-complete p-groups, J. Algebra $\mathbf{5 5 5}$ (2020), 406-424.

[13] R.S. Pierce, Homomorphisms of primary abelian groups, Topics in Abelian Groups, Chicago, Illinois (1963), 215-310.

[14] J. Hausen and J.A. Johnson, Separability of sufficiently projective p-groups as reflected in their endomorphism rings, J. Algebra 69 (1981), 270-280. 
Department of Mathematics and Mechanics, Tomsk State University, 634050 Tomsk, RUSSIA

E-mail address: cheklov@math.tsu.ru; a.r.che@yandex.ru

Institute of Mathematics and Informatics, Bulgarian Academy of Sciences, 1113

Sofia, Bulgaria

E-mail address: danchev@math.bas.bg; pvdanchev@yahoo.com

Technological University, Dublin, Dublin 7, Ireland

E-mail address: brendan.goldsmith@TUDublin.ie 